\title{
Synthesis of Ferrocenyl Oxindole Compounds with Potential Anticancer Activity
}

\author{
Bárbara V. Silva, ${ }^{* a}$ Núbia M. Ribeiro, ${ }^{a}$ Angelo C. Pinto, ${ }^{a}$ Maria D. Vargas ${ }^{b}$ and Luiz C. Dias ${ }^{c}$ \\ ${ }^{a}$ Instituto de Química-CT, Bloco A, Universidade Federal do Rio de Janeiro, Cidade Universitária, \\ 21945-970 Rio de Janeiro-RJ, Brazil \\ ${ }^{b}$ Instituto de Química, Universidade Federal Fluminense, Campus do Valonguinho, Centro, \\ 24020-150 Niterói-RJ, Brazil
}

'Institute of Chemistry, University of Campinas, UNICAMP, P.O. Box 6154, 13084-971 Campinas-SP, Brazil

\begin{abstract}
Compostos oxindol-ferrocenos com potencial atividade anticâncer foram preparados a partir de reações de oxindóis substituídos e carboxaldeído-ferroceno na presença de $\mathrm{KOH}$ como catalisador. Os produtos foram caracterizados por dados espectroscópicos incluindo RMN de ${ }^{1} \mathrm{H}$ e ${ }^{13} \mathrm{C}$, infravermelho e espectrometria de massas. As configurações $E$ e $Z$ foram estabelecidas por experimentos de nOe ou NOESY.
\end{abstract}

A series of ferrocenyl oxindoles with potential anticancer activity were prepared from the reactions of substituted oxindoles and ferrocenylcarboxyaldehyde in the presence of $\mathrm{KOH}$ as catalyst. The products were characterized by spectral data including ${ }^{1} \mathrm{H}$ and ${ }^{13} \mathrm{C} \mathrm{NMR}$, IR and mass spectrometry. The $E$ and $Z$ configurations were established by nOe or NOESY experiments.

Keywords: ferrocenyl oxindoles, oxindoles, ferrocenecarboxyaldehyde

\section{Introduction}

The ferrocenyl $(\mathrm{Fc})$ group has been incorporated to the structure of a number of biologically active molecules resulting in increased anticancer ${ }^{1,2}$ and antimalarial ${ }^{3-5}$ activity, amongst others. Addition, alkylation, acylation, condensation-dehydration, coupling and nucleophilic substitution reactions have been employed to link this group to a variety of compounds..$^{2-4,6,7}$ The mechanisms of the cytotoxicity of ferrocene derivatives have been investigated. ${ }^{8}$ Osella et al. ${ }^{9}$ proposed that the cytotoxic activity of ferrocenium salts is due to their ability to generate oxygen active species that induce oxidative DNA damage. Kondapi et al. ${ }^{10,11}$ have shown that ferrocene derivatives inhibit topoisomerase II, a major molecular target for a number of DNA-binding anticancer drugs.

Indolin-2-ones have been shown to exhibit antitumor activity by inhibiting receptor tyrosine kinases VEGF-R, PDGF-RB or CDK. ${ }^{12}$ The indolin-2-one $\mathbf{1}$ (sunitinib) has been approved by the U.S. Food and Drug Administration for the treatment of metastatic renal cell cancer

*e-mail: barbara.iq@gmail.com and gastrointestinal stromal tumors ${ }^{13,14}$ (Figure 1). Raghunathan et al. ${ }^{15}$ reported recently the synthesis of the ferrocenyl oxindole 2a with $(E)$-configuration from the reaction of oxindole and ferrocenecarboxyaldehyde $(\mathrm{FcC}(\mathrm{O}) \mathrm{H})$ in ethanol, in the presence of catalytic amounts of piperidine.
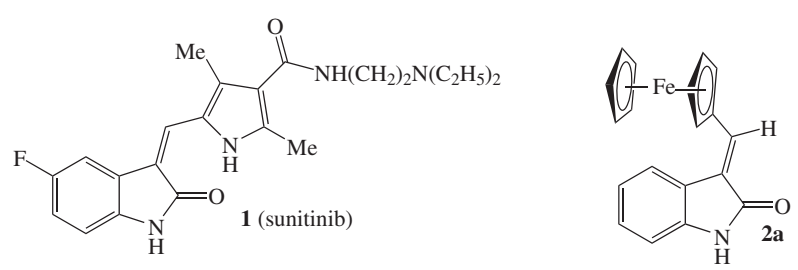

Figure 1. Structure of sunitinib (1) and ferrocenyl oxindole (2a) with (E)-configuration.

We describe herein the condensation reactions of several oxindole derivatives with $\mathrm{FcC}(\mathrm{O}) \mathrm{H}$ in the presence of $\mathrm{KOH}$ in $\mathrm{EtOH}$ from which good to excellent yields of the products with $E$ and $Z$ configurations were obtained (Scheme 1). ${ }^{16}$ The products were characterized by mass spectrometry and infrared, ${ }^{1} \mathrm{H}$ and ${ }^{13} \mathrm{C}$ NMR spectroscopies, including nOe and NOESY experiments for the determination of the double-bond geometry. 
<smiles>[R]c1c([R])c([R4])c2c(c1[R])CC(=O)N2[R]</smiles><smiles>O[Mg]O</smiles>

2, $\mathrm{R}^{1}=\mathrm{R}^{2}=\mathrm{R}^{3}=\mathrm{R}^{4}=\mathrm{R}^{5}=\mathrm{H}$

3, $\mathrm{R}^{1}=\mathrm{R}^{3}=\mathrm{R}^{4}=\mathrm{R}^{5}=\mathrm{H} ; \mathrm{R}^{2}=\mathrm{I}$

4, $\mathrm{R}^{1}=\mathrm{R}^{2}=\mathrm{R}^{3}=\mathrm{R}^{5}=\mathrm{H} ; \mathrm{R}^{4}=\mathrm{Cl}$

$5, \mathrm{R}^{1}=\mathrm{R}^{3}=\mathrm{R}^{5}=\mathrm{H} ; \mathrm{R}^{2}=\mathrm{R}^{4}=\mathrm{Cl}$

6, $\mathrm{R}^{2}=\mathrm{R}^{3}=\mathrm{R}^{5}=\mathrm{H} ; \mathrm{R}^{1}=\mathrm{R}^{4}=\mathrm{Cl}$

7, $\mathrm{R}^{1}=\mathrm{R}^{3}=\mathrm{R}^{5}=\mathrm{H} ; \mathrm{R}^{2}=\mathrm{R}^{4}=\mathrm{Br}$

8, $\mathrm{R}^{2}=\mathrm{R}^{4}=\mathrm{R}^{5}=\mathrm{H} ; \mathrm{R}^{1}=\mathrm{R}^{3}=\mathrm{Br}$

9, $\mathrm{R}^{1}=\mathrm{R}^{2}=\mathrm{R}^{3}=\mathrm{R}^{4}=\mathrm{H} ; \mathrm{R}^{5}=\mathrm{Bn}$

10, $R^{1}=R^{2}=R^{3}=R^{5}=H ; R^{4}=M e$

Scheme 1. Synthesis of ferrocenyl oxindoles.

Preliminary studies of the reaction of oxindole with $\mathrm{FcC}(\mathrm{O}) \mathrm{H}$ (Table 1) were carried out to assess the catalytic potential of various bases.

Table 1. Condensation of oxindole and $\mathrm{FcC}(\mathrm{O}) \mathrm{H}$ in ethanol, at room temperature, in the presence of various bases

\begin{tabular}{llccc}
\hline Entry & Base & time & \multicolumn{2}{c}{ Yield $(\%)$} \\
\cline { 3 - 5 } & & & $\mathbf{2 a}(E)$ & $\mathbf{2 b}(Z)$ \\
\hline 1 & $\mathrm{KOH}(100 \mathrm{mg})$ & $15 \mathrm{~min}$ & 95 & 5 \\
2 & $\mathrm{~K}_{2} \mathrm{CO}_{3}(100 \mathrm{mg})$ & $15 \mathrm{~min}$ & 80 & 18 \\
3 & Piperidine $(0.025 \mathrm{~mL})$ & $15 \mathrm{~min}$ & 67 & 30 \\
4 & Morpholine $(0.025 \mathrm{~mL})$ & $15 \mathrm{~min}$ & 75 & 20 \\
5 & Et $_{3} \mathrm{~N}(0.025 \mathrm{~mL})$ & $96 \mathrm{~h}$ & 33 & 13 \\
\hline
\end{tabular}

Except for $\mathrm{Et}_{3} \mathrm{~N}$, all the bases employed led to good conversions. However, formation of products with both $E$ and $Z$ configurations depend on the base employed. The use of $\mathrm{KOH}$ in $\mathrm{EtOH}$ gave the best yield and selectivity for the $E$-isomer $\mathbf{2 a}$ (entry 1 ). In the presence of piperidine (entry 3 ) we were able to isolate the $E$-isomer $\mathbf{2 a}$ in $67 \%$ yield together with a $30 \%$ isolated yield for the corresponding $Z$-isomer $\mathbf{2 b}$. The geometries of isomers $\mathbf{2 a}$ and $\mathbf{2 b}$ were determined by nOe experiments and NOESY interactions. The NOESY experiment for $\mathbf{2 a}$ shows a correlation between $\mathrm{H} 4$ and $\mathrm{H} 2$ ' of the cyclopentadienyl ring, thus revealing an $(E)$-configuration for this isomer. In the case of $\mathbf{2 b}$, we have observed a $2.15 \%$ intensity enhancement of the peak corresponding to $\mathrm{H} 4$ upon irradiation of $\mathrm{H} 8$, which confirms the $(Z)$-configuration (Figure 2).

The condensation reactions of a series of substituted oxindoles with $\mathrm{FcC}(\mathrm{O}) \mathrm{H}$ were then investigated in the
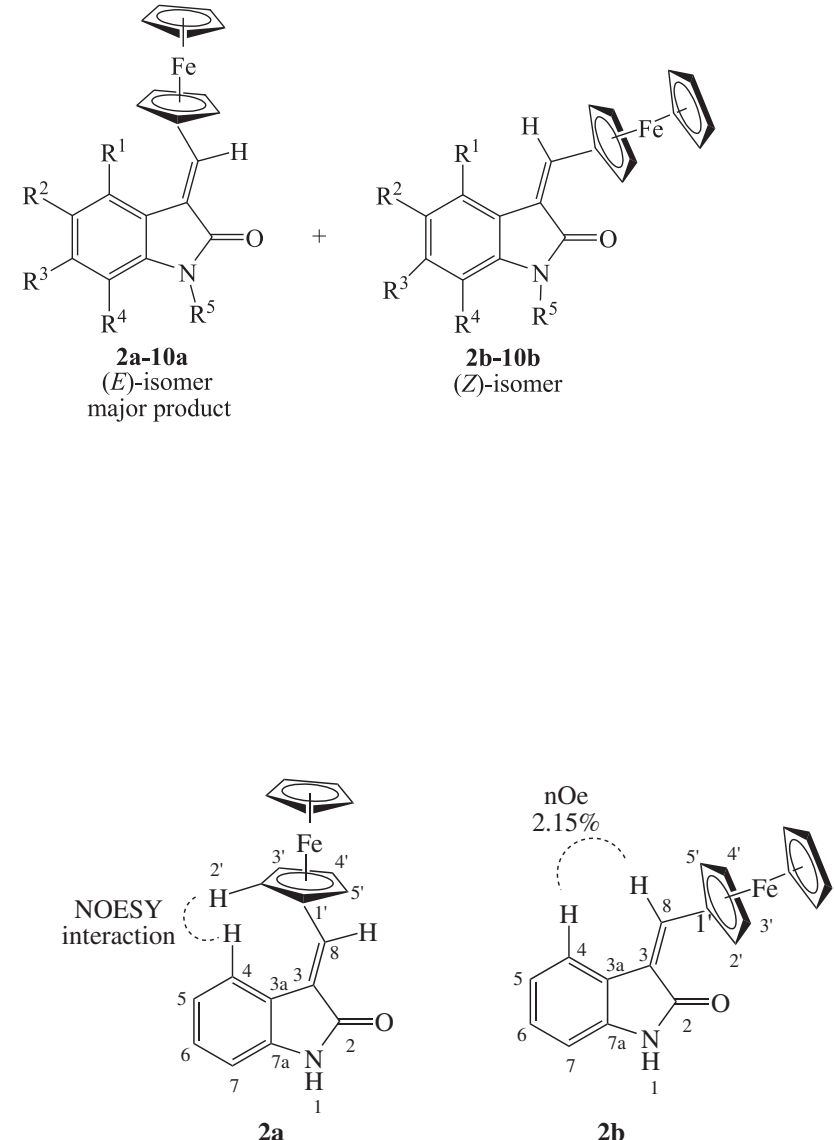

Figure 2. Hydrogen correlations that confirm the structures of compounds $\mathbf{2 a}$ and $\mathbf{2 b}$.

presence of $\mathrm{KOH}$ in EtOH. Products and yields are summarized in Table 2.

The reactions of monosubstituted oxindoles $\mathbf{3}, \mathbf{4}$ and 10 (entries 1, 2 and 8, respectively) gave good conversions and the same product distribution as that of oxindole, with $E$-products being formed as the major isomers in yields above $90 \%$. Product yields decreased with further ring substitution, and were also sensitive to the relative position of the substituents: whereas the reactions of 5,7-dichloro and 5,7-dibromo oxindoles ( $\mathbf{5}$ and $\mathbf{7}$, entries 3 and 5 , respectively) gave both isomers albeit in lower yields than oxindole, those of the 4,7-dichloro and 4,6-dibromo oxindoles ( $\mathbf{6}$ and $\mathbf{8}$, entries 4 and 6 , respectively) resulted only in the formation of the $E$-isomer in around $45 \%$ yields.

As shown in Table 3, in all cases, the olefinic hydrogen H8 of $E$-isomer (2a-10a) appear at higher frequency than for the $Z$-isomer (2b-10b), due to the diamagnetic anisotropy effect of the carbonyl oxygen (C2) on $\mathrm{H} 8$ of $E$-isomers 2a-10a. The same effect was observed for $\mathrm{H}^{\prime}{ }^{\prime}$ and $\mathrm{H}^{\prime}{ }^{\prime}$ of the $Z$-isomers $\mathbf{2 b - 1 0 b}$. H8 chemical shifts for compounds 6a and 8a at D8.40 and 8.34 pm, respectively, confirm the (E)-configuration. 
Table 2. Condensation reactions of oxindole derivatives with $\mathrm{FcC}(\mathrm{O}) \mathrm{H}$ in the presence of $\mathrm{KOH}$ as catalyst

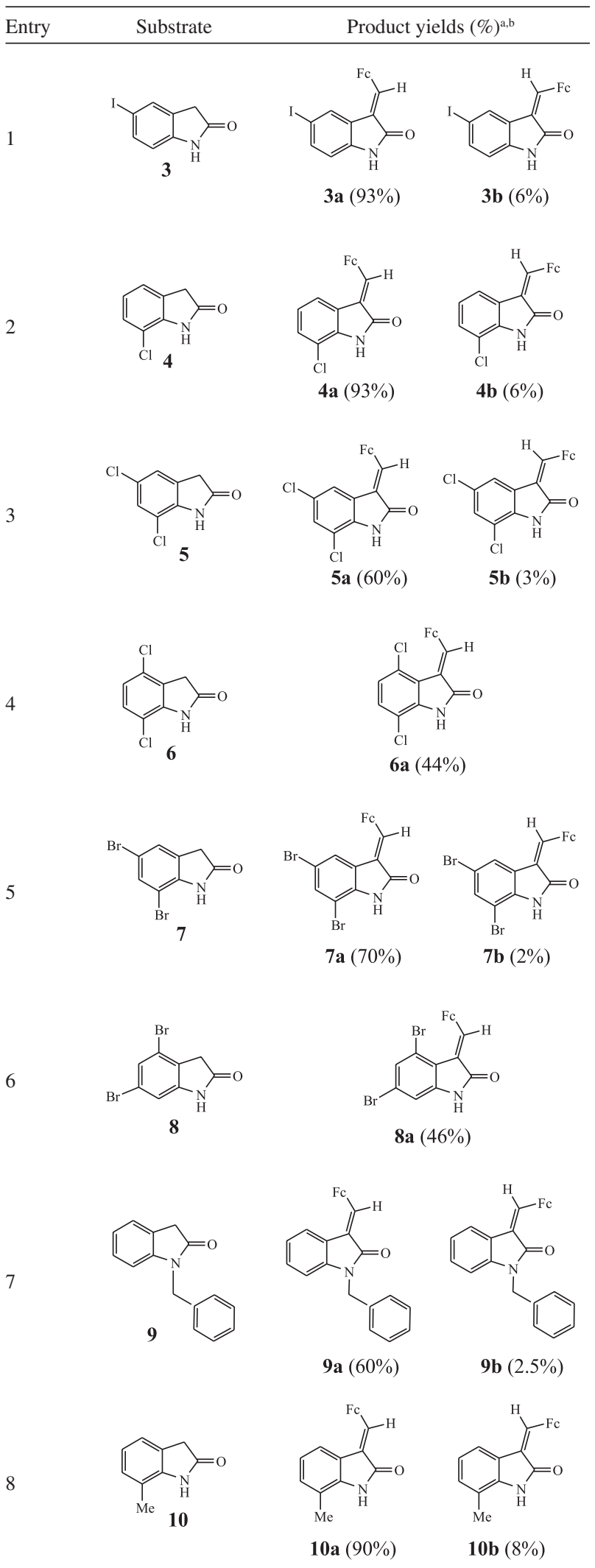

$\overline{\text { a yields are for isolated products after flash column chromatography on }}$ silica-gel; ${ }^{\mathrm{b}} \mathrm{Fc}=$ ferrocenyl.
Table 3. Chemical shifts of $\mathrm{H} 8$, and $\mathrm{H} 2$ ' and $\mathrm{H} 5$ ' for compounds $\mathbf{2 a}, \mathbf{b}$ to $10 \mathbf{a}, \mathbf{b}$

\begin{tabular}{lcccc}
\hline \multirow{2}{*}{ Compound } & \multicolumn{2}{c}{$\mathrm{H} 8$} & & \multicolumn{2}{c}{$\mathrm{H} 2{ }^{\prime}$ and $\mathrm{H} 5$} \\
\cline { 2 - 3 } \cline { 5 - 5 } & $\mathbf{a}$ & $\mathbf{b}$ & $\mathbf{a}$ & $\mathbf{b}$ \\
\hline $\mathbf{2}$ & $7.54(\mathrm{~s})$ & $7.41(\mathrm{~s})$ & $4.68(\mathrm{t}, J 1.7 \mathrm{~Hz})$ & $5.34(\mathrm{t}, J 1.9 \mathrm{~Hz})$ \\
$\mathbf{3}$ & $7.71(\mathrm{~s})$ & $7.39(\mathrm{~s})$ & $4.75(\mathrm{br}, \mathrm{s})$ & $5.33(\mathrm{t}, J 1.9 \mathrm{~Hz})$ \\
$\mathbf{4}$ & $7.71(\mathrm{~s})$ & $7.43(\mathrm{~s})$ & $4.74(\mathrm{br}, \mathrm{s})$ & $5.34(\mathrm{t}, J 1.9 \mathrm{~Hz})$ \\
$\mathbf{5}$ & $7.79(\mathrm{~s})$ & $7.44(\mathrm{~s})$ & $4.75(\mathrm{t}, J 1.7 \mathrm{~Hz})$ & $4.81(\mathrm{t}, J 1.6 \mathrm{~Hz})$ \\
$\mathbf{6}$ & $8.40(\mathrm{~s})$ & - & $5.39(\mathrm{~m})$ & - \\
$\mathbf{7}$ & $7.76(\mathrm{~s})$ & $7.43(\mathrm{~s})$ & $4.66(\mathrm{~m})$ & $5.34(\mathrm{t}, J 1.6 \mathrm{~Hz})$ \\
$\mathbf{8}$ & $8.34(\mathrm{~s})$ & - & $5.11(\mathrm{t}, J 1.9 \mathrm{~Hz})$ & - \\
$\mathbf{9}$ & $7.76(\mathrm{~s})$ & $7.30(\mathrm{~s})$ & $4.77(\mathrm{t}, J 1.7 \mathrm{~Hz})$ & $5.36(\mathrm{t}, J 1.9 \mathrm{~Hz})$ \\
$\mathbf{1 0}$ & $7.67(\mathrm{br}, \mathrm{s})$ & $7.39(\mathrm{~s})$ & $4.80(\mathrm{br}, \mathrm{s})$ & $5.34(\mathrm{t}, J 1.8 \mathrm{~Hz})$ \\
\hline
\end{tabular}

\section{Conclusion}

In conclusion we have described a simple, fast and efficient synthetic route to ferrocenyl oxindoles, potential model compounds for chemical and pharmacological studies.

\section{Acknowledgments}

The authors thank the Brazilian agencies National Counsel for Technological and Scinentific Development (CNPq), State of Rio de Janeiro Research Foundation (FAPERJ), State of São Paulo Research Foundation (FAPESP) and Pronex-FAPERJ for financial support. We also thank Prof. Carol H. Collins (Institute of Chemistry, UNICAMP) for helpful suggestions about English grammar and style.

\section{References}

1. Top, S.; Tang, J.; Vessières, A.; Carrez, D.; Provot, C.; Jaouen, G.; Chem. Commun. 1996, 955.

2. Hillard, E. A.; Pigeon, P.; Vessières, A.; Amatore, C.; Jaouen, G.; Dalton Trans. 2007, 5073.

3. Domarle, O.; Blampain, G.; Agnaniet, H.; Nzadiyabi, T.; Lebibi, J.; Brocard, J.; Maciejewski, L.; Biot, C.; Georges, A. J.; Millet, P.; Antimicrob. Agents Chemother. 1998, 42, 540.

4. Atteke, C.; Ndong, J. M. M.; Aubouy, A.; Maciejewski, L.; Brocard, J.; Lebibi, J.; Deloron, P. J.; Antimicrob. Chemother. 2003, 51, 1021.

5. Blackie, M. A. L.; Chibale, K.; Met. Based Drugs 2008, 2008: ID 495123.

6. Biot, C.; Delhaes, L.; Maciejewski, L. A.; Mortuaire, M.; Camus, D.; Dive, D.; Brocard, J. S.; Eur. J. Med. Chem. 2000, 35,707 . 
7. Torres, J. C.; Pilli, R. A.; Vargas, M. D.; Violante, F. A.; Garden, S. J.; Pinto, A. C.; Tetrahedron 2002, 58, 4487.

8. Köpf-Maier, Köpf, H.; Neuse, E. W.; J. Cancer Res. Clin. Oncol. 1984, 108, 336.

9. Osella, D.; Ferrali, M.; Zanello, P.; Laschi, F.; Fontani, M.; Nervi, C.; Cavigiolio, G.; Inorg. Chim. Acta 2000, 42, 306.

10. Gopal, Y. N. V.; Jayaraju, D.; Kondapi, K.; Arch. Biochem. Biophys. 2000, 376, 229.

11. Kondapi, A. K.; Satyanarayana, N.; Sai krishna, A.D.; Arch. Biochem. Biophys. 2006, 450, 123.

12. Bramson, H. N.; Corona, J.; Davis, S. T.; Dickerson, S. H.; Edelstein, M.; Frye, S. V.; Gampe, R. T., Jr.; Harris, P. A.; Hassell, A.; Holmes, W. D.; Hunter, R. N.; Lackey, K. E.; Lovejoy, B.; Luzzio, M. J.; Montana, V.; Rocque, W. J.; Rusnak, D.; Shewchuk, L.; Veal, J. M.; Walker, D. H.; Kuyper, L. F.; J. Med. Chem. 2001, 44, 4339; Bogoyevitch, M. A; Fairlie, D. P.; Drug Discov. Today 2007, 12, 622; Cerchiaro, G.; Ferreira, A. M. C.; J. Braz. Chem. Soc. 2006, 17, 1473.

13. Sun, L.; Liang, C.; Shirazian, S.; Zhou, Y.; Miller, T. Cui, J.; Fukuda, J. Y.; Chu, J-Y; Nematalla, A.; Wang, X.; Chen, H.;
Sistla, A.; Luu, T. C.; Tang, F.; Wei, J. Tang., C.; J. Med. Chem. 2003, 46, 1116.

14. Roskoski, R. Jr.; Biochem. Biophys. Res. Commun. 2007, 356, 323.

15. Jayashankaran, J.; Durga, R.; Manian, R. S.; Raghunathan, R.; Synth. Commun. 2006, 36, 979; Babu, A. R. S.; Raghunathan, R.; Tetrahedron Lett. 2008, 49, 4487.

16. General experimental procedure: A mixture of oxindole $(0.2 \mathrm{mmol})$, ferrocenecarboxyaldehyde $(0.22 \mathrm{mmol})$, ethanol $(5 \mathrm{~mL})$ and $100 \mathrm{mg}$ of $\mathrm{KOH}$ was allowed to stir at $\mathrm{rt}$ for $15 \mathrm{~min}$ (2) or under reflux for $1 \mathrm{~h} \mathrm{(3-10)}$. The progress of the reactions was monitored by TLC. After total consumption of the substrate, the crude reaction mixture was extracted with ethyl acetate and washed with water. The organic layer was separated, dried $\left(\mathrm{Na}_{2} \mathrm{SO}_{4}\right)$ and concentrated in vacuo. The $E$ and $Z$ products were separated by silica gel column chromatography (using hexane/ ethyl acetate as eluent).

Received: July 15, 2008 Web Release Date: August 27, 2008

FAPESP helped in meeting the publication costs of this article. 


\section{Synthesis of Ferrocenyl Oxindole Compounds with Potential Anticancer Activity}

\section{Bárbara V. Silva, ${ }^{a}$ Núbia M. Ribeiro, ${ }^{a}$ Angelo C. Pinto, ${ }^{a}$ Maria D. Vargas ${ }^{b}$ and Luiz C. Dias ${ }^{c}$}

${ }^{a}$ Instituto de Química-CT, Bloco A, Universidade Federal do Rio de Janeiro, Cidade Universitária, 21945-970 Rio de Janeiro-RJ, Brazil

${ }^{b}$ Instituto de Química, Universidade Federal Fluminense, Campus do Valonguinho, Centro, 24020-150 Niterói-RJ, Brazil

${ }^{c}$ Institute of Chemistry, University of Campinas, UNICAMP, P.O. Box 6154, 13084-971 Campinas-SP, Brazil

Table S1. ${ }^{1} \mathrm{H}$ NMR data for compounds $\mathbf{2 a - 2 b}$ to $\mathbf{1 0 a}-\mathbf{1 0 b}$

\begin{tabular}{|c|c|c|c|c|c|c|c|c|}
\hline \multirow[t]{2}{*}{ Carbon } & \multicolumn{8}{|c|}{${ }^{1} \mathrm{H}$ chemical shifts $\left(\delta / \mathrm{ppm}, 250 \mathrm{MHz}, \mathrm{CDCl}_{3}\right)$} \\
\hline & $2 a$ & $2 \mathbf{b}$ & $3 \mathrm{a}^{*}$ & $3 b^{*}$ & $4 a^{*}$ & $4 b^{*}$ & $5 \mathbf{5 a}$ & $5 \mathbf{b}$ \\
\hline 1 & $9.28(\mathrm{~s})$ & $7.97(\mathrm{~s})$ & $8.32(\mathrm{~s})$ & $7.89(\mathrm{~s})$ & $8.54(\mathrm{~s})$ & $7.84(\mathrm{~s})$ & $8.21(\mathrm{~s})$ & $8.41(\mathrm{~s})$ \\
\hline 2 & - & - & - & - & - & - & - & - \\
\hline 3 & - & - & - & - & - & - & - & - \\
\hline 4 & $7.54(\mathrm{~d}, J 7.5 \mathrm{~Hz})$ & $7.49(\mathrm{~d}, J 7.5 \mathrm{~Hz})$ & $8.31(\mathrm{br}, \mathrm{s})$ & $7.77(\mathrm{~d}, J 1.6 \mathrm{~Hz})$ & $7.17(\mathrm{~d}, J 8.0 \mathrm{~Hz})$ & $7.19(\mathrm{~d}, J 8.0 \mathrm{~Hz})$ & $7.21(\mathrm{~d}, J 1.6 \mathrm{~Hz})$ & $7.52(\mathrm{~d}, J 1.4 \mathrm{~Hz})$ \\
\hline 5 & $\begin{array}{c}7.10(\mathrm{td}, J 7.5 \\
1.0 \mathrm{~Hz})\end{array}$ & $\begin{array}{c}7.21(\mathrm{td}, J 7.5 \\
1.1 \mathrm{~Hz})\end{array}$ & - & - & $6.89(\mathrm{t}, J 8.0 \mathrm{~Hz})$ & $6.96(\mathrm{t}, J 8.0 \mathrm{~Hz})$ & - & - \\
\hline 6 & $\begin{array}{c}6.86(\mathrm{dd}, J 7.5 \\
1.0 \mathrm{~Hz})\end{array}$ & $\begin{array}{c}7.02(\mathrm{td}, J 7.5 \\
1.1 \mathrm{~Hz})\end{array}$ & $6.69(\mathrm{~d}, J 8.2 \mathrm{~Hz})$ & $\begin{array}{c}7.50(\mathrm{dd}, J 8.0 \\
1.6 \mathrm{~Hz})\end{array}$ & $7.83(\mathrm{~d}, J 8.0 \mathrm{~Hz})$ & $7.37(\mathrm{~d}, J 8.0 \mathrm{~Hz})$ & $7.88(\mathrm{~d}, J 1.6 \mathrm{~Hz})$ & $7.48(\mathrm{~d}, J 1.4 \mathrm{~Hz})$ \\
\hline 7 & $6.81(\mathrm{~d}, J 7.5 \mathrm{~Hz})$ & $6.84(\mathrm{~d}, J 7.5 \mathrm{~Hz})$ & $7.53(\mathrm{~d}, J 8.2 \mathrm{~Hz})$ & $6.63(\mathrm{~d}, J 8.0 \mathrm{~Hz})$ & - & - & - & - \\
\hline 8 & $7.54(\mathrm{~s})$ & $7.41(\mathrm{~s})$ & $7.71(\mathrm{~s})$ & $7.39(\mathrm{~s})$ & $7.71(\mathrm{~s})$ & $7.43(\mathrm{~s})$ & $7.79(\mathrm{~s})$ & $7.44(\mathrm{~s})$ \\
\hline 9 & - & - & - & - & - & - & - & - \\
\hline 10 & - & - & - & - & - & - & - & - \\
\hline 1 ' & - & - & - & - & - & - & - & - \\
\hline $2^{\prime}-5$ & $4.68(\mathrm{t}, J 1.7 \mathrm{~Hz})$ & $5.34(\mathrm{t}, J 1.9 \mathrm{~Hz})$ & $4.75(\mathrm{br}, \mathrm{s})$ & $5.33(\mathrm{t}, J 1.9 \mathrm{~Hz})$ & $4.74(\mathrm{br}, \mathrm{s})$ & $5.34(\mathrm{t}, J 1.9 \mathrm{~Hz})$ & $4.75(\mathrm{t}, J 1.7 \mathrm{~Hz})$ & $4.81(\mathrm{t}, J 1.6 \mathrm{~Hz})$ \\
\hline $3^{\prime}-4{ }^{\prime}$ & $4.49(\mathrm{t}, J 1.7 \mathrm{~Hz})$ & $4.60(\mathrm{t}, J 1.9 \mathrm{~Hz})$ & $4.66(\mathrm{br}, \mathrm{s})$ & $4.64(\mathrm{t}, J 1.9 \mathrm{~Hz})$ & $4.60(\mathrm{br}, \mathrm{s})$ & $4.64(\mathrm{t}, J 1.9 \mathrm{~Hz})$ & $4.69(\mathrm{t}, J 1.7 \mathrm{~Hz})$ & $4.62(\mathrm{t}, J 1.6 \mathrm{~Hz})$ \\
\hline \multirow[t]{2}{*}{$\mathrm{Cp}$} & $4.14(\mathrm{~s})$ & $4.20(\mathrm{~s})$ & $4.26(\mathrm{~s})$ & $4.21(\mathrm{~s})$ & $4.21(\mathrm{~s})$ & $4.20(\mathrm{~s})$ & $4.26(\mathrm{~s})$ & $4.28(\mathrm{~s})$ \\
\hline & $6 a$ & $7 a^{*}$ & $7 b^{*}$ & $8 a^{*}$ & $9 a$ & $9 \mathrm{~b}$ & $10 \mathrm{a}$ & $10 \mathrm{~b}$ \\
\hline 1 & $8.44(\mathrm{~s})$ & $8.06(\mathrm{br}, \mathrm{s})$ & $7.71(\mathrm{~s})$ & $9.91(\mathrm{~s})$ & - & - & $8.87(\mathrm{~s})$ & $8.21(\mathrm{~s})$ \\
\hline 2 & - & - & - & - & - & - & - & - \\
\hline 3 & - & - & - & - & - & - & - & - \\
\hline 4 & - & $8.06(\mathrm{br}, \mathrm{s})$ & $7.20(\mathrm{~d}, J 1.4 \mathrm{~Hz})$ & - & $7.99(\mathrm{~d}, J 7.6 \mathrm{~Hz})$ & $7.50(\mathrm{~d}, J 7.0 \mathrm{~Hz})$ & $7.82(\mathrm{~d}, J 2.0 \mathrm{~Hz})$ & $7.34(\mathrm{~d}, J 7.4 \mathrm{~Hz})$ \\
\hline 5 & $7.08(\mathrm{~d}, J 7.9 \mathrm{~Hz})$ & - & - & $7.15(\mathrm{~d}, J 1.4 \mathrm{~Hz})$ & $7.15(\mathrm{t}, J 7.6 \mathrm{~Hz})$ & $\begin{array}{c}7.15(\mathrm{td}, J 7.0 \\
1.0 \mathrm{~Hz})\end{array}$ & $6.91-7.06(\mathrm{br}, \mathrm{m})$ & $6.95(\mathrm{t}, J 7.4 \mathrm{~Hz})$ \\
\hline 6 & $6.95(\mathrm{~d}, J 7.9 \mathrm{~Hz})$ & $7.49(\mathrm{~s})$ & $7.36(\mathrm{~d}, J 1.4 \mathrm{~Hz})$ & - & $6.95(\mathrm{t}, J 7.6 \mathrm{~Hz})$ & $\begin{array}{c}7.00(\mathrm{td}, J 7.0 \\
1.0 \mathrm{~Hz})\end{array}$ & $6.91-7.06(\mathrm{br}, \mathrm{m})$ & $7.04(\mathrm{~d}, J 7.4 \mathrm{~Hz})$ \\
\hline 7 & - & - & - & $6.85(\mathrm{~d}, J 1.4 \mathrm{~Hz})$ & $6.72(\mathrm{~d}, J 7.6 \mathrm{~Hz})$ & $6.72(\mathrm{~d}, J 7.0 \mathrm{~Hz})$ & - & - \\
\hline 8 & $8.40(\mathrm{~s})$ & $7.76(\mathrm{~s})$ & $7.43(\mathrm{~s})$ & $8.34(\mathrm{~s})$ & $7.76(\mathrm{~s})$ & $7.30(\mathrm{~s})$ & 7.67 (br, s) & $7.39(\mathrm{~s})$ \\
\hline 9 & - & - & - & - & $5.00(\mathrm{~s})$ & 5.00 & $2.35(\mathrm{~s})$ & $2.30(\mathrm{~s})$ \\
\hline 10 & - & - & - & - & $7.32(\mathrm{~m})$ & $7.32(\mathrm{~m})$ & - & - \\
\hline 1 ' & - & - & - & - & - & - & - & - \\
\hline $2^{\prime}-3{ }^{\prime}$ & $5.39(\mathrm{~m})$ & $4.66(\mathrm{~m})$ & $5.34(\mathrm{t}, J 1.6 \mathrm{~Hz})$ & $5.11(\mathrm{t}, J 1.9 \mathrm{~Hz})$ & $4.77(\mathrm{t}, J 1.7 \mathrm{~Hz})$ & $5.36(\mathrm{t}, J 1.9 \mathrm{~Hz})$ & $4.80(\mathrm{br}, \mathrm{s})$ & $5.34(\mathrm{t}, J 1.8 \mathrm{~Hz})$ \\
\hline $4^{\prime}-5$, & $4.69(\mathrm{~m})$ & $4.66(\mathrm{~m})$ & $4.68(\mathrm{t}, J 1.6 \mathrm{~Hz})$ & $4.49(\mathrm{t}, J 1.9 \mathrm{~Hz})$ & $4.58(\mathrm{t}, J 1.7 \mathrm{~Hz})$ & $4.58(\mathrm{t}, J 1.9 \mathrm{~Hz})$ & $4.59(\mathrm{br}, \mathrm{s})$ & $4.49(\mathrm{t}, J 1.8 \mathrm{~Hz})$ \\
\hline $\mathrm{Cp}$ & $4.22(\mathrm{~s})$ & $4.25(\mathrm{~s})$ & $4.21(\mathrm{~s})$ & $4.07(\mathrm{~s})$ & $4.25(\mathrm{~s})$ & $4.21(\mathrm{~s})$ & $4.24(\mathrm{~s})$ & $4.19(\mathrm{~s})$ \\
\hline
\end{tabular}

*e-mail: barbara.iq@gmail.com 
Table S2. ${ }^{13} \mathrm{C}$ NMR data for compounds $\mathbf{2 a - 2 b}$ to $\mathbf{1 0 a - 1 0 b}$

\begin{tabular}{|c|c|}
\hline \multicolumn{2}{|r|}{${ }^{13} \mathrm{C}$ chemical shifts $\left(\delta / \mathrm{ppm}, 62.5 \mathrm{MHz}, \mathrm{CDCl}_{3}\right)$} \\
\hline $2 a$ & $\begin{array}{l}170.2,141.1,137.3,128.2,123.3,122.4,122.3,121.1,109.8, \\
78.3,71.1,69.6\end{array}$ \\
\hline $2 b^{* * *}$ & $\begin{array}{l}168.2,138.8,138.4,127.4,125.7,121.5,118.2,109.3,104.7 \\
73.6,72.4,68.9\end{array}$ \\
\hline \multicolumn{2}{|l|}{$3 a^{*}$} \\
\hline \multicolumn{2}{|l|}{$3 \mathbf{b}$} \\
\hline $4 a^{*}$ & $\begin{array}{l}169.4,140.2,138.1,127.9,124.1,122.6,122.3,120.8,115.2 \\
71.9,71.5,70.0\end{array}$ \\
\hline \multicolumn{2}{|l|}{$4 b$} \\
\hline $5 \mathbf{a}$ & $\begin{array}{l}168.9,142.3,136.4,127.2,127.2,125.0,121.4,120.9,115.4 \text {, } \\
72.6,71.8,70.2\end{array}$ \\
\hline \multicolumn{2}{|l|}{$\mathbf{5 b}$} \\
\hline \multicolumn{2}{|l|}{$6 \mathbf{a}$} \\
\hline \multicolumn{2}{|l|}{$7 \mathbf{a}$} \\
\hline \multicolumn{2}{|l|}{$7 \mathbf{b}$} \\
\hline $8 a^{*}$ & $\begin{array}{l}167.6,143.2,142.1,128.0,121.4,120.5,119.7,115.4,111.7 \\
74.0,72.8,70.1\end{array}$ \\
\hline 9a & $\begin{array}{l}168.4,142.3,138.3,136.3,128.7,128.3,127.4,127.3,122.7,122.5 \\
122.1,121.6,108.9,108.9,73.1,71.3,69.9\end{array}$ \\
\hline \multicolumn{2}{|l|}{$9 b$} \\
\hline \multicolumn{2}{|l|}{$10 \mathbf{a}$} \\
\hline $10 b$ & \\
\hline
\end{tabular}

Table S3. Infrared data for compounds $\mathbf{2 a - 2 b}$ to $\mathbf{1 0 a - 1 0 b}$

\begin{tabular}{|c|c|}
\hline \multicolumn{2}{|r|}{$\mathrm{IR}(\mathrm{KBr}) \mathrm{v}_{\max } / \mathrm{cm}^{-1}$} \\
\hline $2 \mathbf{a}$ & $\begin{array}{l}3362,3122,3066,3020,2902,2838,1693,1621,1608,1465, \\
1338,1220\end{array}$ \\
\hline $2 b$ & $\begin{array}{l}3434,3133,3064,3021,2923,2850,1695,1619,1600,1473, \\
1211\end{array}$ \\
\hline 3a & $3435,3145,3101,8248,1697,1618,1600,1442,1223$ \\
\hline 3b & $3447,3141,3097,2918,1688,1615,1596,1473,1201$ \\
\hline $4 \mathbf{a}$ & $\begin{array}{l}3367,3170,3135,3079,3002,2879,2802,1695,1623,1602, \\
1481,1438,1330,1205,1187\end{array}$ \\
\hline $4 b$ & $\begin{array}{l}3446,3161,3003,2880,1685,1260,1586,1481,1439,1369, \\
1189\end{array}$ \\
\hline $5 \mathbf{a}$ & $3445,3132,3073,2971,1688,1594,1577,1468,1208,1162$ \\
\hline $5 \mathbf{b}$ & $3419,3112,2921,2850,1697,1600,1573,1448,1301,1151$ \\
\hline $6 \mathbf{a}$ & $3481,3135,2921,2850,1692,1614,1578,1466,1323,1146$ \\
\hline $7 \mathbf{a}$ & $\begin{array}{l}3441,3135,3066,2956,2848,1697,1621,1599,1584,1447, \\
1227,1132\end{array}$ \\
\hline $7 \mathbf{b}$ & $\begin{array}{l}3357,3127,3005,2924,2854,1709,1689,1591,1463,1443, \\
1207,1155\end{array}$ \\
\hline $8 \mathbf{a}$ & $3428,3114,3081,2958,2921,2852,1689,1604,1581,1178$ \\
\hline 9a & $\begin{array}{l}3442,3130,3137,3079,1695,1623,1604,1604,1483,1330 \text {, } \\
1187,1130\end{array}$ \\
\hline $9 \mathrm{~b}$ & $3442,3128,3052,2956,2917,1695,1616,1604,1484,1168$ \\
\hline $10 \mathbf{a}$ & $\begin{array}{l}3461,3357,3144,2918,2833,1690,1608,1594,1336,1218 \text {, } \\
1189\end{array}$ \\
\hline 10b & $\begin{array}{l}3466,3419,3138,2917,2849,1686,1626,1596,1369,1206 \text {, } \\
1044\end{array}$ \\
\hline
\end{tabular}

2a

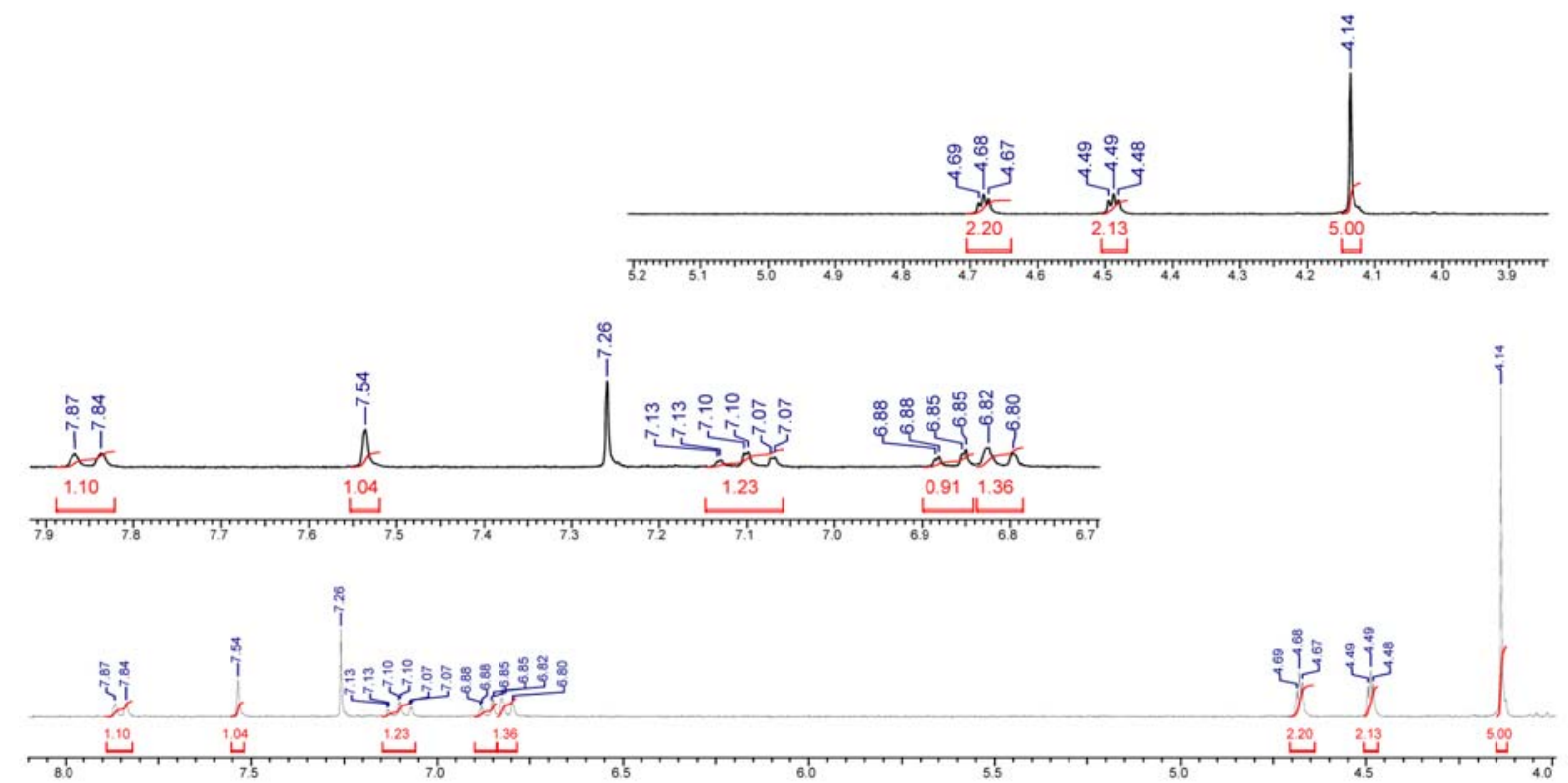

Figure S1. ${ }^{1} \mathrm{H}$ NMR of 2 a. 
$2 a$

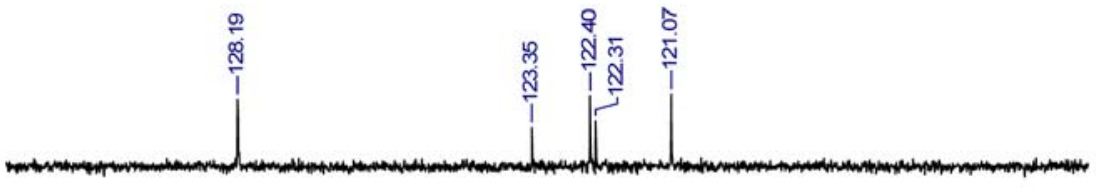

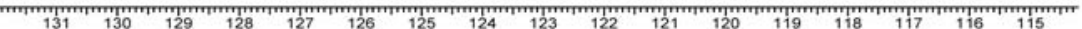

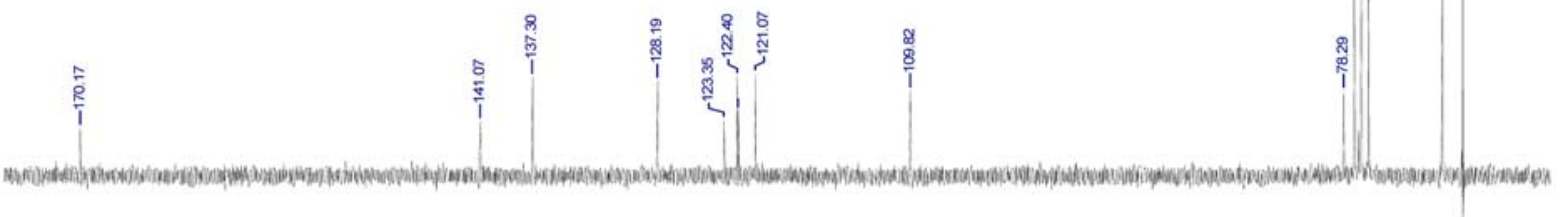

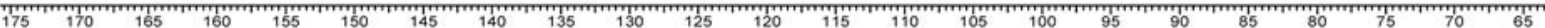

Figure S2. ${ }^{13} \mathrm{C}$ NMR of $\mathbf{2 a}$.

$2 b$

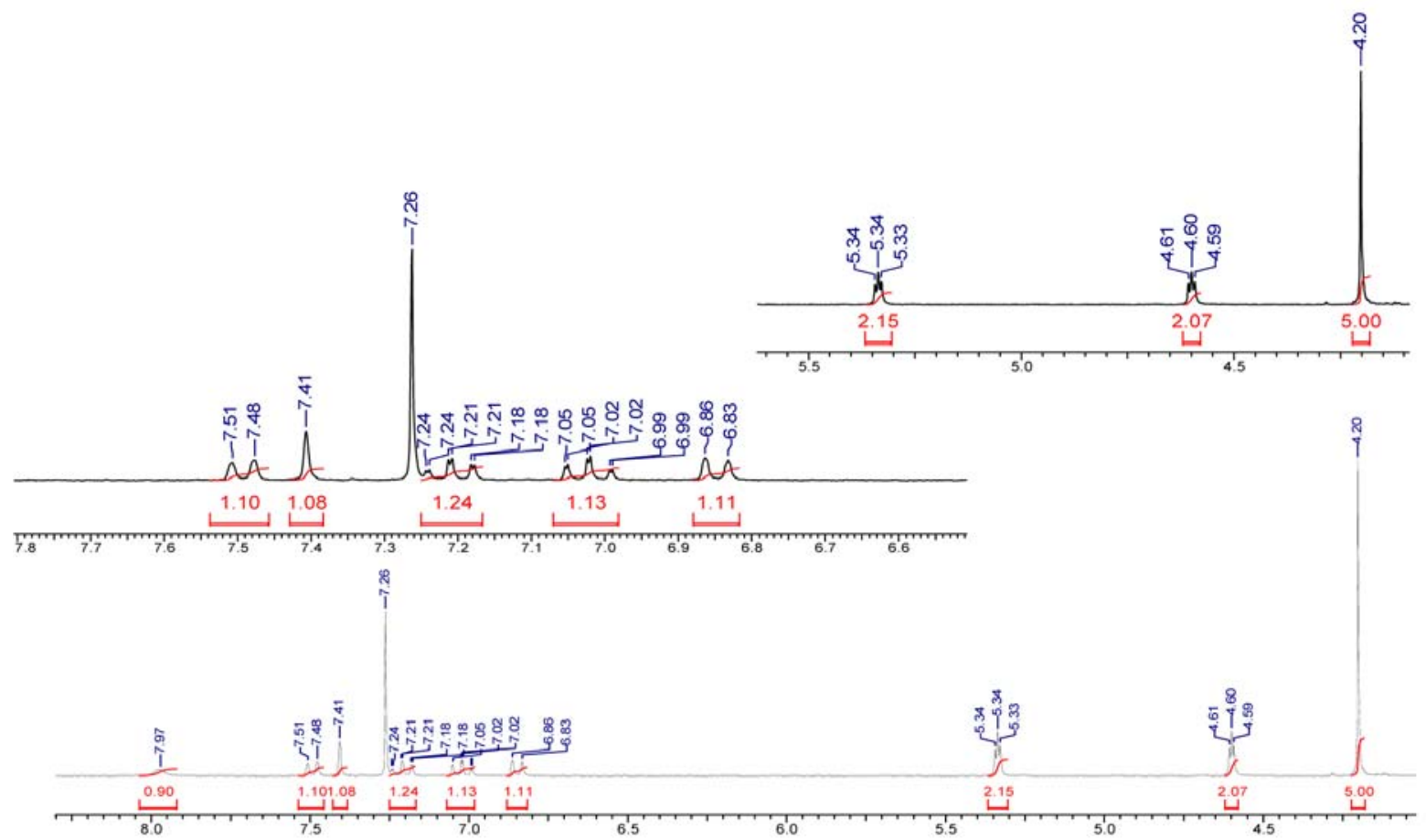

Figure S3. ${ }^{1} \mathrm{H}$ NMR of $\mathbf{2 b}$. 


\section{2b}

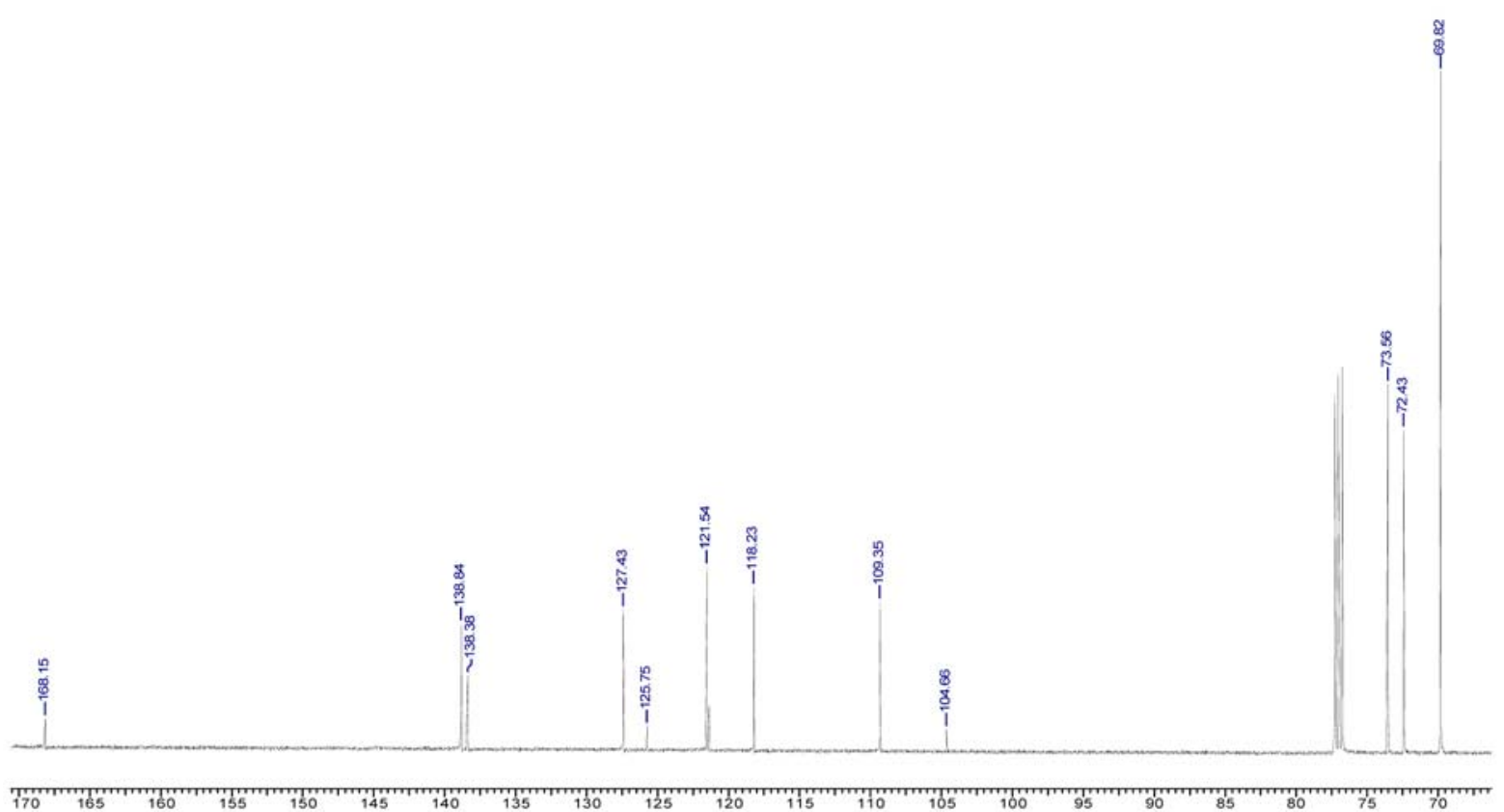

Figure S4. ${ }^{13} \mathrm{C}$ NMR of $\mathbf{2 b}$.

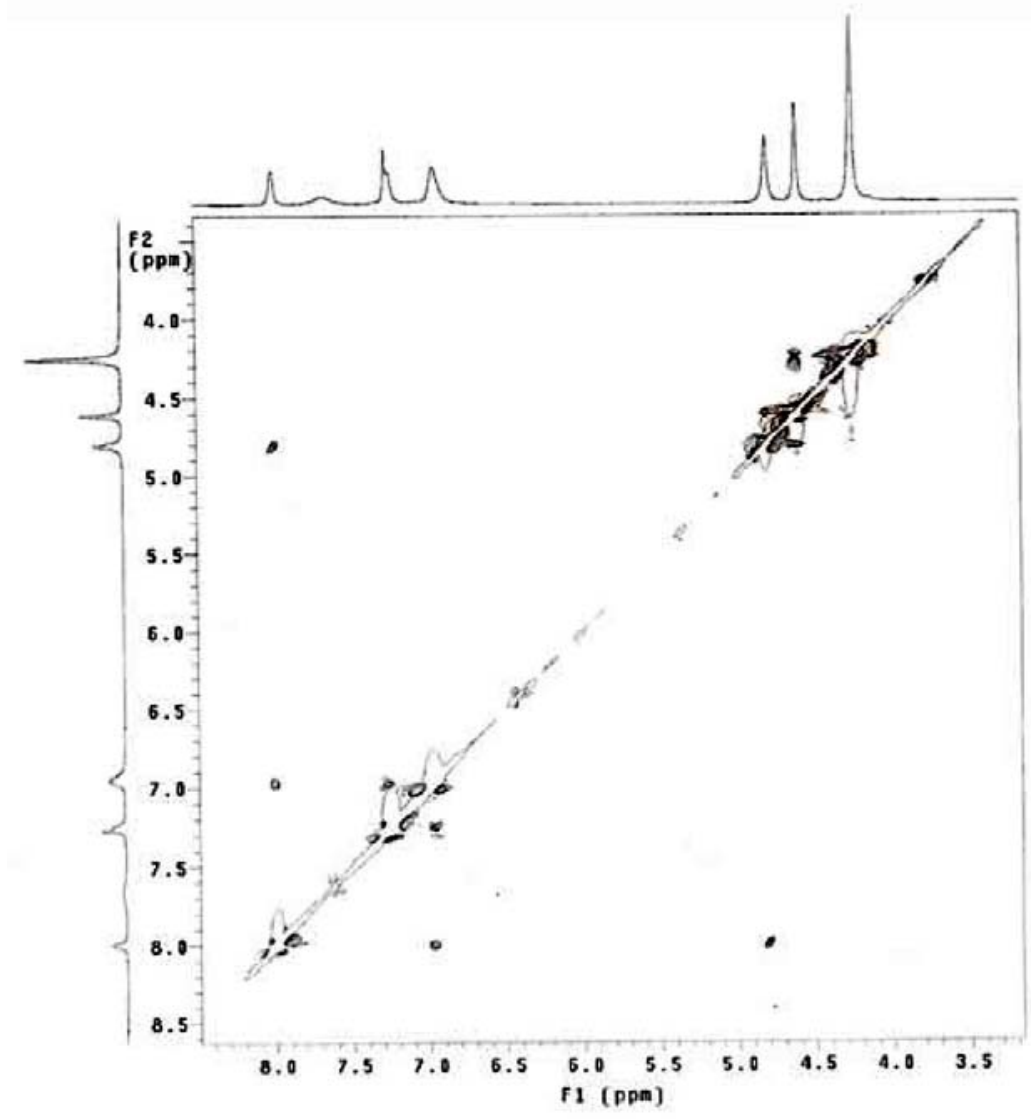

Figure S5. NOESY of 2a. 


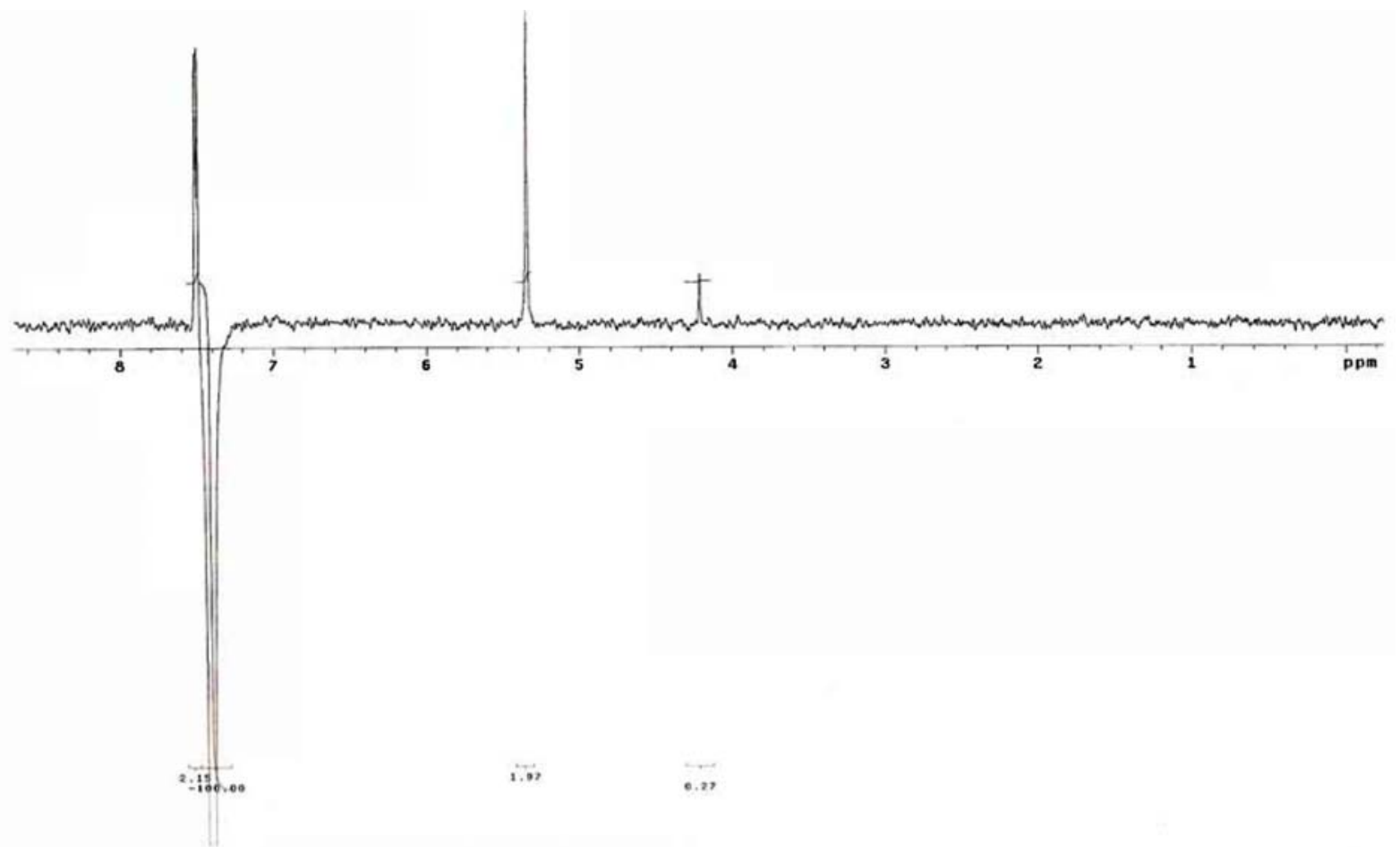

Figure S6. nOe of $\mathbf{2 b}$

$3 a$

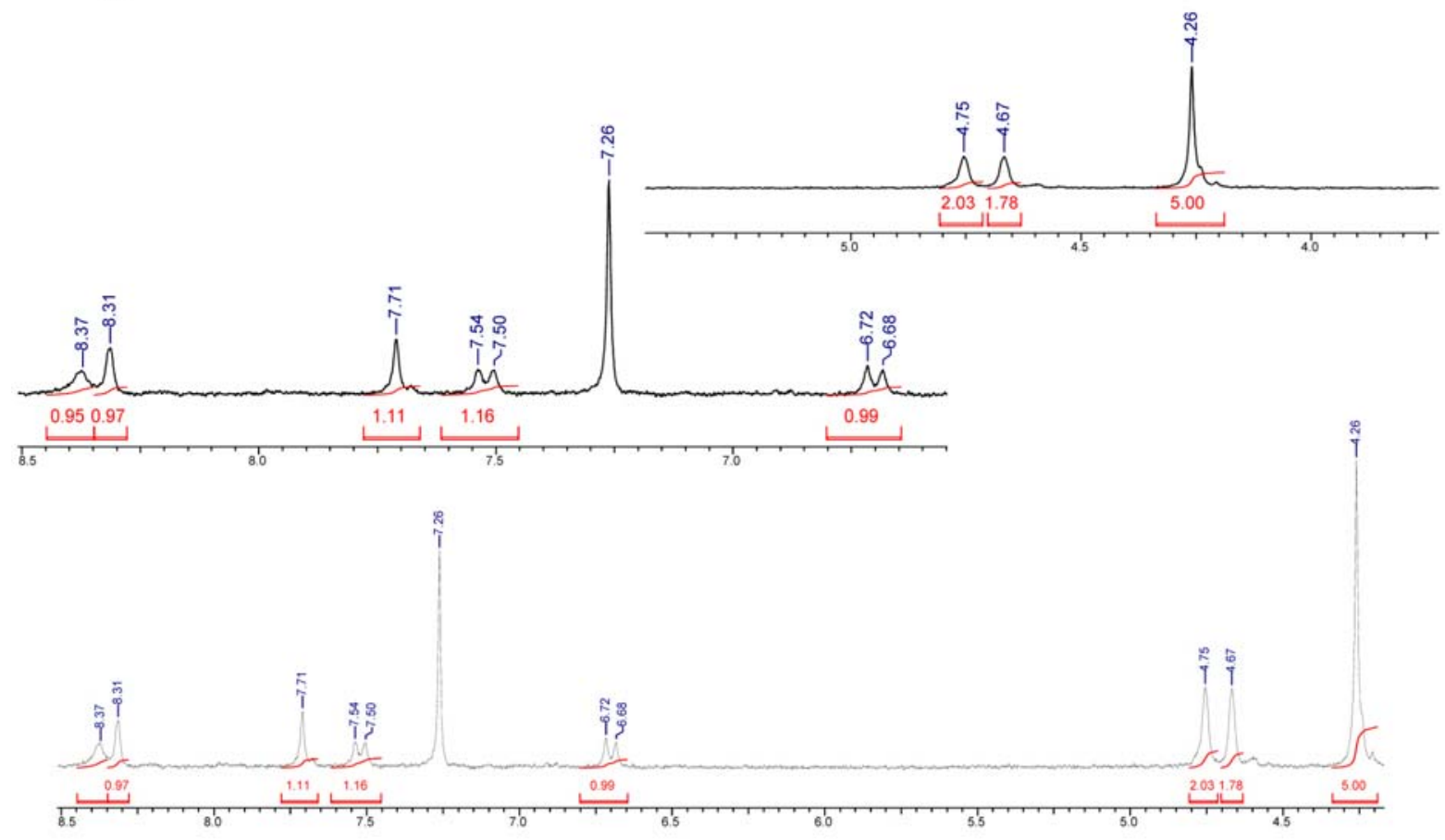

Figure S7. ${ }^{1} \mathrm{H}$ NMR of $\mathbf{3 a}$. 
3b

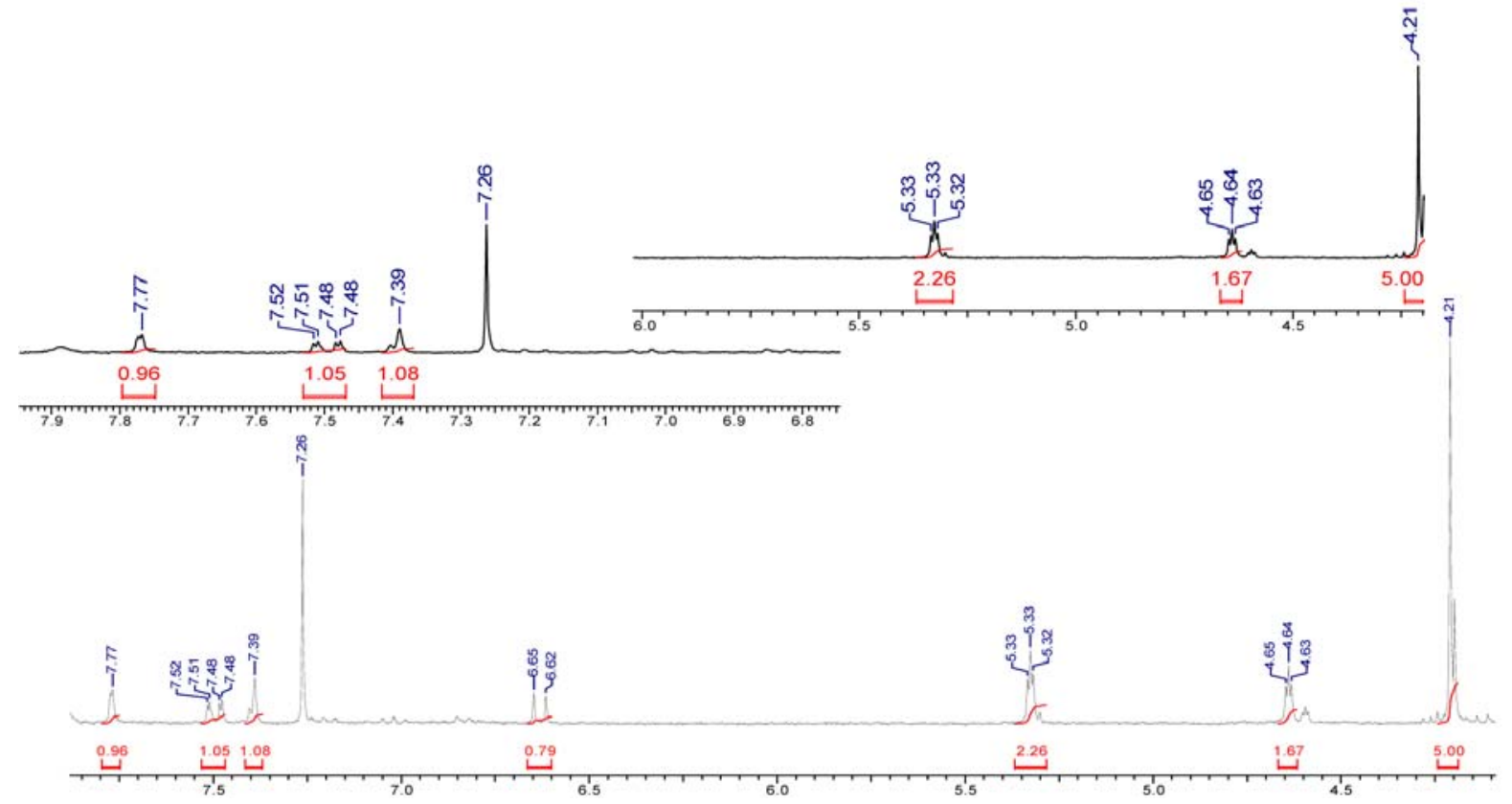

Figure S8. ${ }^{1} \mathrm{H}$ NMR of $\mathbf{3 b}$.

$4 a$

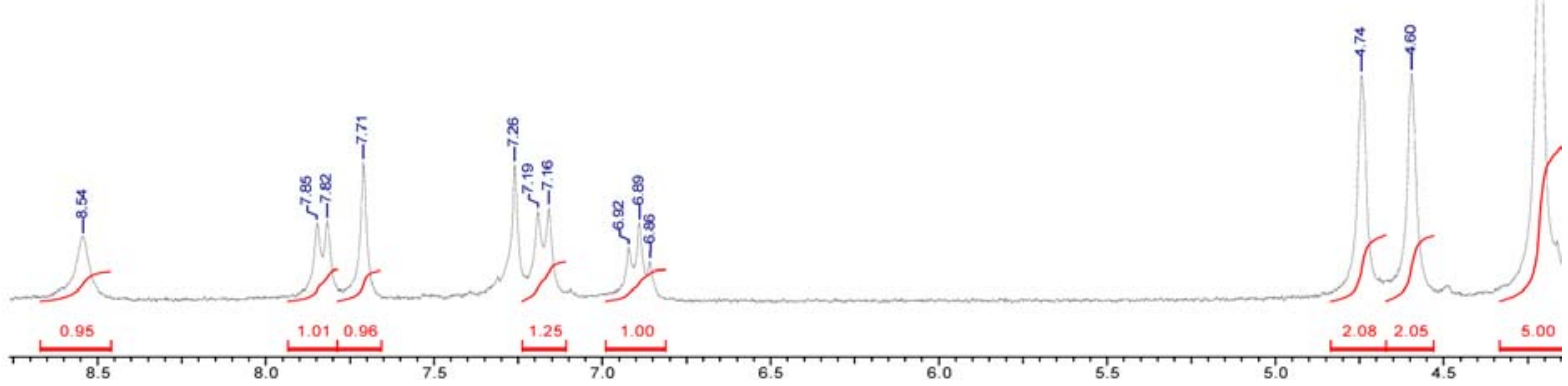

Figure S9. ${ }^{1} \mathrm{H}$ NMR of $\mathbf{4 a}$ 
$4 a$

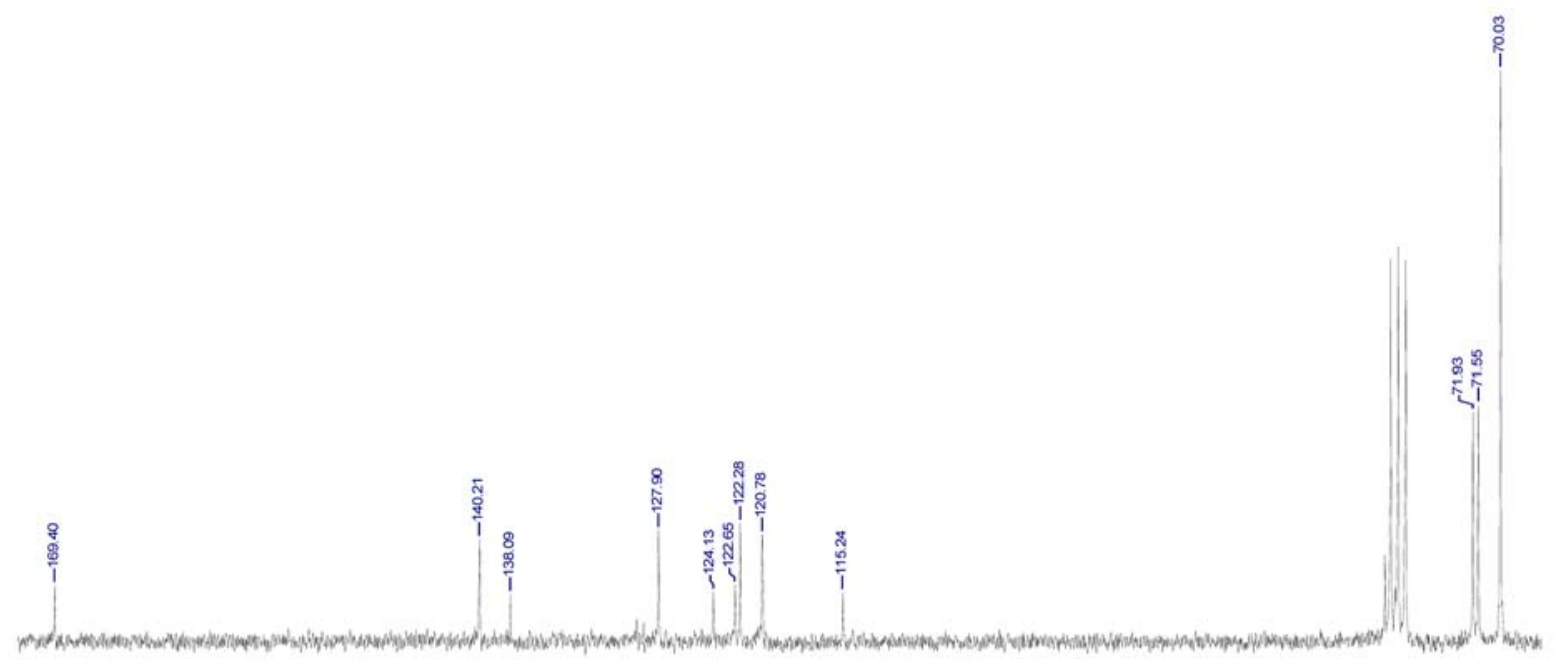

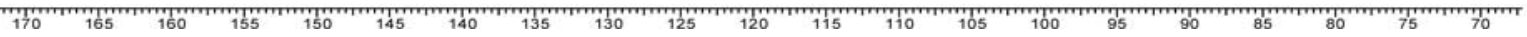

Figure S10. ${ }^{13} \mathrm{C}$ NMR of $4 a$.

4b

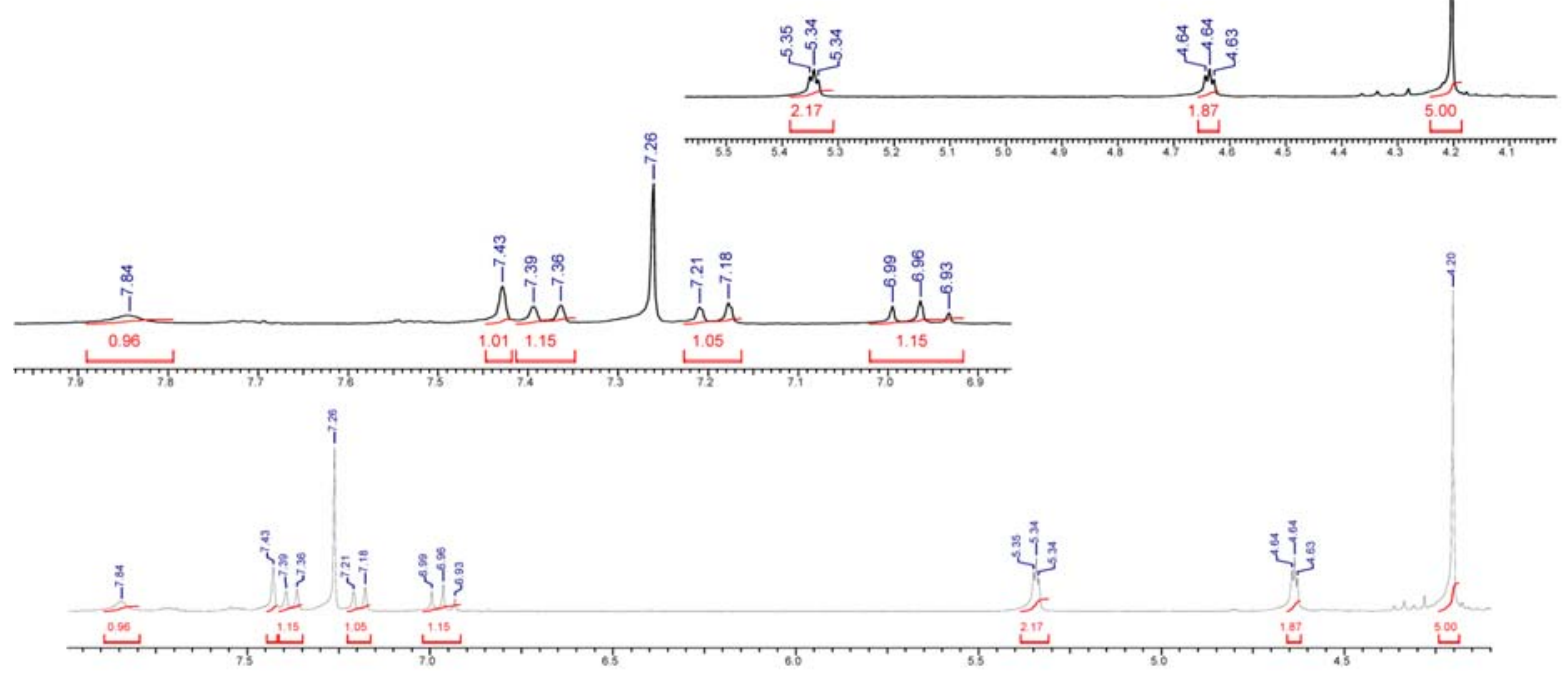

Figure S11. ${ }^{1} \mathrm{H}$ NMR of $\mathbf{4 b}$. 
7b

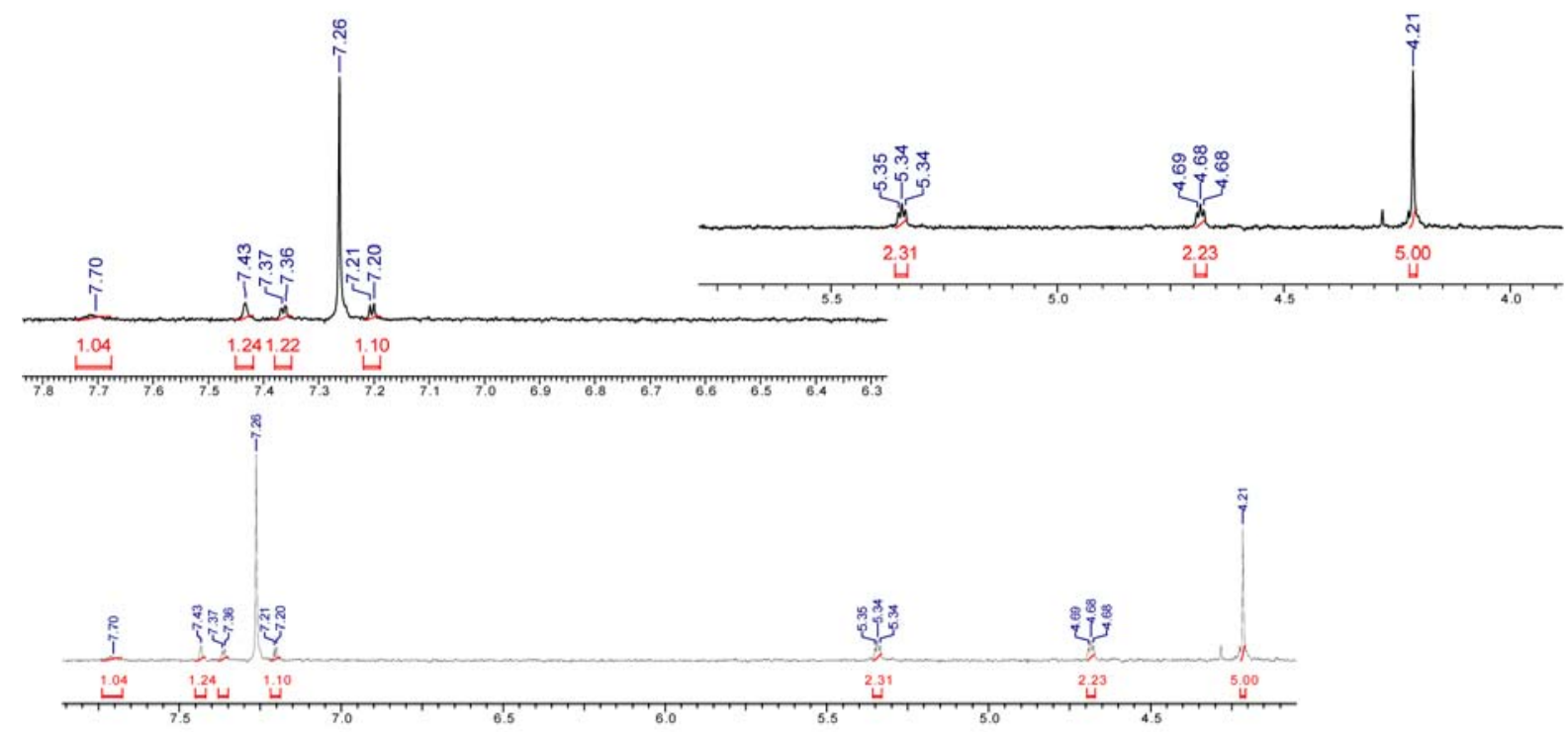

Figure S12. ${ }^{1} \mathrm{H}$ NMR of $7 \mathbf{b}$.

9b
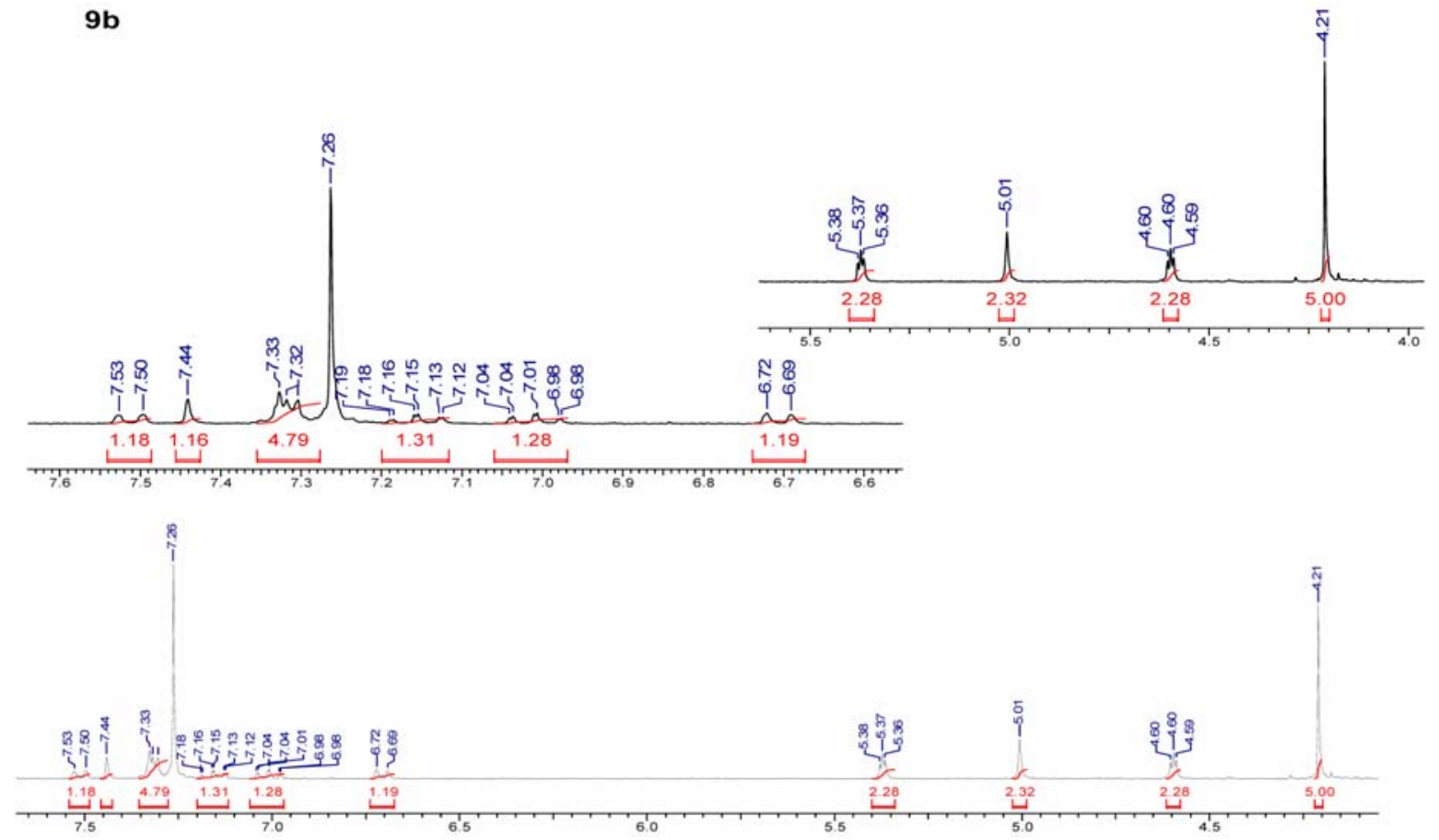

Figure S13. ${ }^{1} \mathrm{H}$ NMR of $9 \mathbf{b}$. 
$10 \mathrm{~b}$
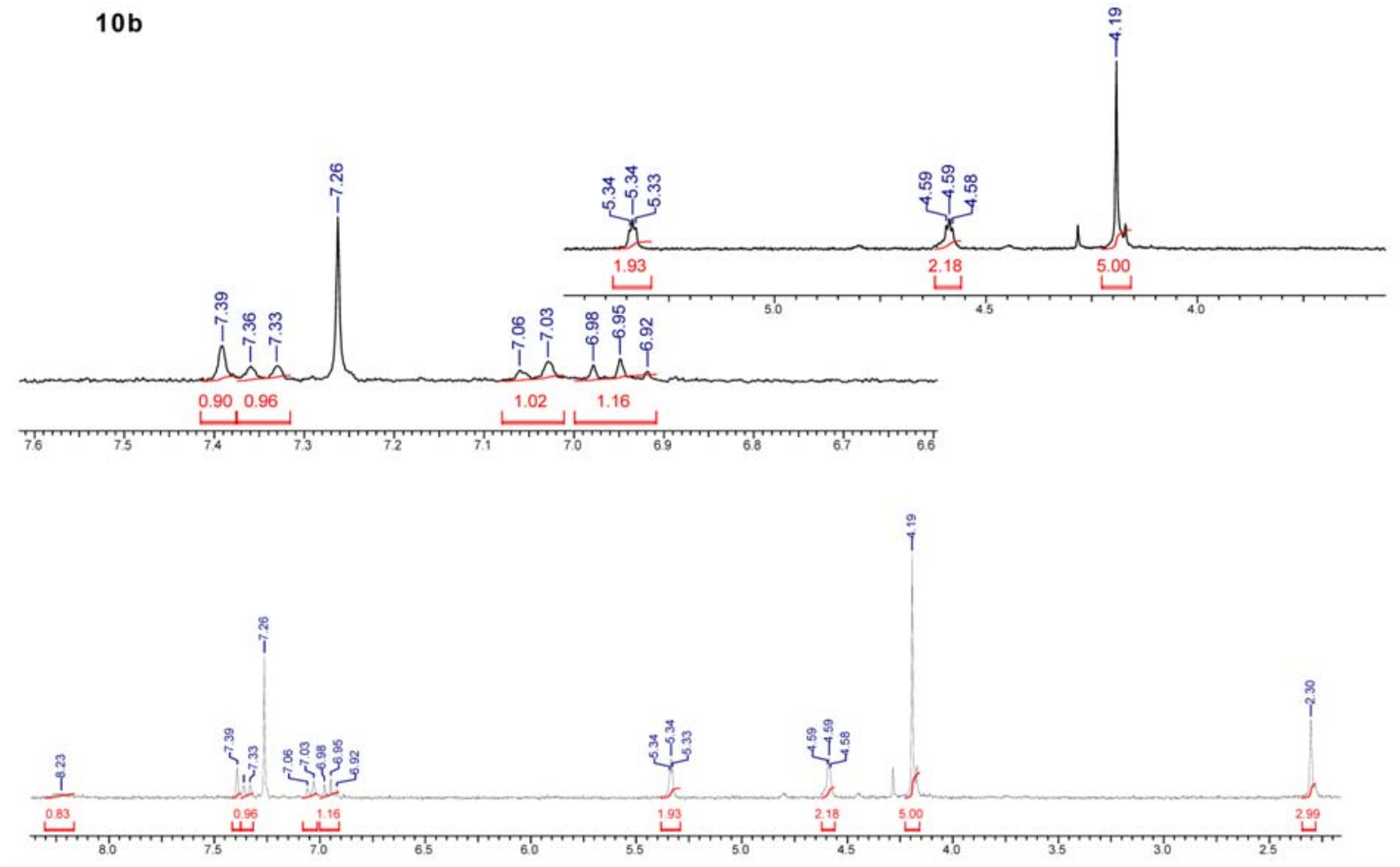

Figure S14. ${ }^{1} \mathrm{H}$ NMR of $\mathbf{1 0 b}$. 